\title{
Statistical mechanics of multipartite entanglement
}

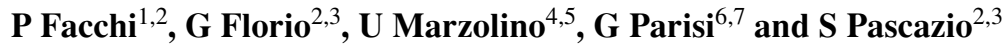 \\ ${ }^{1}$ Dipartimento di Matematica, Università di Bari, I-70125 Bari, Italy \\ ${ }^{2}$ Istituto Nazionale di Fisica Nucleare, Sezione di Bari, I-70126 Bari, Italy \\ ${ }^{3}$ Dipartimento di Fisica, Università di Bari, I-70126 Bari, Italy \\ ${ }^{4}$ Dipartimento di Fisica Teorica, Università di Trieste, Strada Costiera 11, 34014 Trieste, Italy \\ ${ }^{5}$ INFN, Sezione di Trieste, Trieste, Italy \\ ${ }^{6}$ Dipartimento di Fisica, Università di Roma 'La Sapienza', Piazzale Aldo Moro 2, 00185 Roma, \\ Italy, Centre for Statistical Mechanics and Complexity (SMC), CNR-INFM, 00185 Roma, Italy \\ ${ }^{7}$ INFN, Sezione di Roma, 00185 Roma, Italy
}

Received 24 October 2008, in final form 29 October 2008

Published 6 January 2009

Online at stacks.iop.org/JPhysA/42/055304

\begin{abstract}
We characterize the multipartite entanglement of a system of $n$ qubits in terms of the distribution function of the bipartite purity over all balanced bipartitions. We search for those (maximally multipartite entangled) states whose purity is minimum for all bipartitions and recast this optimization problem into a problem of statistical mechanics.
\end{abstract}

PACS numbers: 03.67.Mn, 03.65.Ud, 89.75.-k, 03.67.-a

(Some figures in this article are in colour only in the electronic version)

Entanglement is a consequence of the quantal superposition principle and therefore of the linearity of Hilbert spaces, and embodies the impossibility of factorizing a given state of a total quantum system in terms of the states of its constituents. The notion of entanglement plays a central role in the development of novel resources and techniques in computing, communication and information processing. There is a widespread literature about the characterization, quantification and properties of bipartite entanglement, i.e. the entanglement of two subsystems [1,2]. The landscape of multipartite entanglement is less understood but very widely investigated [3-6]. The main difficulty is that there is no unique way of quantifying it: more so, different definitions often do not agree with each other, because they adopt different strategies and capture different features of this inherently quantum phenomenon.

It would be desirable to understand how to properly characterize multipartite entanglement, e.g. by identifying a few key properties that can account for its overall features. The quantification and evaluation of global entanglement measures bears serious computational difficulties, because states endowed with large entanglement typically involve exponentially many coefficients. Multipartite entanglement has therefore the typical features of a complex phenomenon, and for this reason we shall follow a precept derived from the study of complex systems, that relies on the idea that complicated phenomena cannot be summarized by a single (or a few) number(s) [7]. We note that in the context of quantum entanglement this 
idea was alluded to in [8]. In this spirit, entanglement can be analyzed in terms of a function: the probability density of the purity of a subsystem over all bipartitions of the total system $[9,10]$. A state has a large multipartite entanglement if it has a large and well-distributed bipartite entanglement. In other words, states with large multipartite entanglement are characterized by a narrow distribution of bipartite entanglement, centered around a large average.

In this paper we shall explore these ideas even further and endeavor to give a statistical mechanical characterization of multipartite entanglement, by recasting the problem in terms of a cost function that will represent the (inverse) global entanglement of a quantum system. We will establish a direct connection between the study of multipartite entanglement and the tools of classical statistical mechanics.

We shall consider an ensemble of $n$ spin-1/2 particles (qubits), whose Hilbert space $\mathcal{H}=\left(\mathbb{C}^{2}\right)^{\otimes n}$ has dimension $N=2^{n}$. We divide this set in two parts, $A$ and $\bar{A}$, made up of $n_{A}$ and $n_{\bar{A}}$ qubits respectively $\left(n_{A}+n_{\bar{A}}=n\right)$. The total Hilbert space is split in the tensor product $\mathcal{H}=\mathcal{H}_{A} \otimes \mathcal{H}_{\bar{A}}$, with dimensions $N_{A}=2^{n_{A}}$ and $N_{\bar{A}}=2^{n_{\bar{A}}}$ respectively $\left(N_{A} N_{\bar{A}}=N\right)$. We also assume with no loss of generality $n_{A} \leqslant n_{\bar{A}}$. We shall consider only pure states and shall not discuss additional phenomena such as bound entanglement $[11,12]$. Let

$$
|\psi\rangle=\sum_{j=0}^{N-1} z_{j}|j\rangle, \quad z_{j} \in \mathbb{C}, \quad \sum_{j=0}^{N-1}\left|z_{j}\right|^{2}=1,
$$

where the computational base $\{|j\rangle\}$ is expressed in terms of the eigenstates of the third Pauli matrices acting on each site. These yield binary sequences belonging to $\{0,1\}^{n}$, whose decimal representation are the indices $j$. For each bipartition $(A, \bar{A})$ we define a bijection $j \leftrightarrow\left(j_{A}, j_{\bar{A}}\right)$, where the integers $j_{A} \in\left[0, N_{A}-1\right]$ and $j_{\bar{A}} \in\left[0, N_{\bar{A}}-1\right]$ are the decimal representation of the subsequences of portion $A$ and $\bar{A}$, respectively. This reflects the factorization $|j\rangle=\left|j_{A}\right\rangle \otimes\left|j_{\bar{A}}\right\rangle$. As bipartite entanglement measure (at fixed bipartition $(A, \bar{A})$ ) we take the purity of subsystem $A$, which is conveniently expressed in terms of the reduced density operators, $\rho_{A}=\operatorname{tr}_{\bar{A}} \rho$ ( $\rho=|\psi\rangle\langle\psi|$ being the total density operator)

$$
\pi_{A}=\operatorname{tr}_{A} \rho_{A}^{2}=\operatorname{tr}_{\bar{A}} \rho_{\bar{A}}^{2}=\sum_{j_{A}, l_{A}, j_{\bar{A}}, l_{\bar{A}}} z_{j_{A}, j_{\bar{A}}} \bar{z}_{l_{A}, j_{\bar{A}}} z_{l_{A}, l_{\bar{A}}} \bar{z}_{j_{A}, l_{\bar{A}}} .
$$

Purity ranges in $\left[1 / N_{A}, 1\right]$ and is 1 if and only if $\rho_{A}$ and $\rho_{\bar{A}}$ are projectors, that is, if the state is (bi)separable, $|\psi\rangle=\left|\psi_{A}\right\rangle \otimes\left|\psi_{\bar{A}}\right\rangle$. Otherwise $|\psi\rangle$ is entangled. $\pi_{A}$ saturates its minimum $1 / N_{A}$ if and only if the reduced density operator of the smaller partition is proportional to the identity matrix $\rho_{A}=\mathbf{1} / N_{A}$. The latter is the case of states $|\psi\rangle$ endowed with maximum bipartite entanglement, at fixed bipartition $(A, \bar{A})$. The distribution function of purity over all bipartitions, $p\left(\pi_{A}\right)$, yields a probability density function characterization of multipartite entanglement: its average measures the mean entanglement of the state when the bipartitions are varied, while its variance quantifies how uniformly bipartite entanglement is distributed among all possible bipartitions.

The coefficients $z_{j}$ in (1) belong to the set

$$
C=\left\{\left.\left(z_{0}, z_{1}, \ldots, z_{N-1}\right) \in \mathbb{C}^{N}\left|\sum_{k}\right| z_{k}\right|^{2}=1\right\} .
$$

Note that $C$ corresponds to the set of normalized vectors $\mathcal{S}=\{\psi \in \mathcal{H} \mid\|\psi\|=1\}$ and is left invariant under the natural action of the unitary group $\mathcal{U}(\mathcal{H})$. A typical state is obtained by a uniform sampling of $\mathcal{S}$. Typical states can be (efficiently) generated by a chaotic dynamics [13, 14]. 


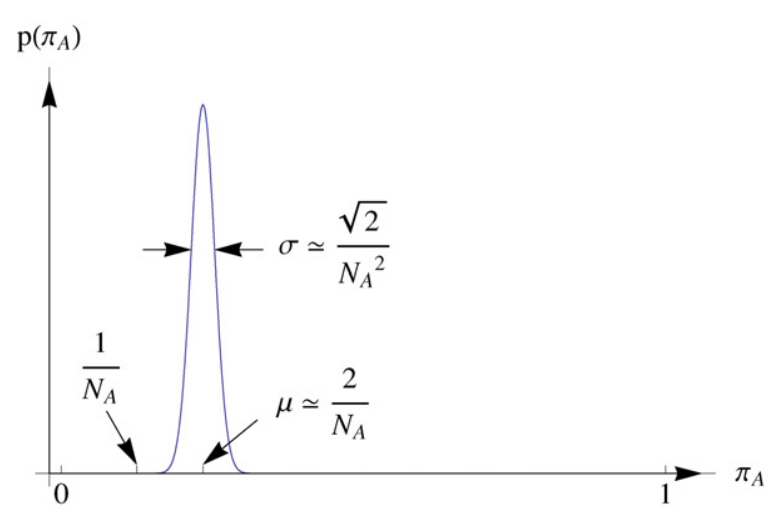

Figure 1. Probability density function $p\left(\pi_{A}\right)$ of a typical state over balanced bipartitions. For large $n$, the average is $2 / N_{A}$ and the standard deviation $\sqrt{2} / N_{A}^{2}\left(N_{A} \simeq \sqrt{N}\right)$. Our objective is to characterize those 'maximally multipartite entangled states' whose average purity is minimum.

In the limit of large $N, \pi_{A}$ 's have a bell-shaped distribution over the bipartitions with mean and variance

$$
\begin{aligned}
& \mu=\left\langle\pi_{A}\right\rangle=\frac{N_{A}+N_{\bar{A}}}{N+1} \\
& \sigma^{2}=\left\langle\left(\pi_{A}-\mu\right)^{2}\right\rangle=\frac{2\left(N_{A}^{2}-1\right)\left(N_{\bar{A}}^{2}-1\right)}{(N+1)^{2}(N+2)(N+3)},
\end{aligned}
$$

respectively $[9,10,15-20]$. The brackets $\langle\cdots\rangle$ denote the average with respect to the unitarily invariant measure over pure states

$$
\mathrm{d} \mu_{C}(z)=\prod_{k} \mathrm{~d} z_{k} \mathrm{~d} \bar{z}_{k} \delta\left(1-\sum_{k}\left|z_{k}\right|^{2}\right),
$$

induced by the Haar measure over $\mathcal{U}(\mathcal{H})$ through the mapping $|\psi\rangle=\sum z_{j}|j\rangle=U\left|\psi_{0}\right\rangle$, for a given state $\left|\psi_{0}\right\rangle[18]$.

It is interesting to note that, for balanced bipartitions $n_{A}=\lfloor n / 2\rfloor$, the average and standard deviation of the distribution simplify to $\mu \simeq 2 / N_{A}, \sigma \simeq \sqrt{2} / N_{A}^{2}$, the approximation becoming quickly very accurate as the number of qubits increases. The behavior of the probability density function $p\left(\pi_{A}\right)$ is sketched in figure 1 . It is worth emphasizing that the typical states are very entangled, but are far from the minimum (on average) by a factor 2; moreover, their distribution function is very narrow (a signature of a good multipartition of entanglement), but has a nonvanishing standard deviation. It is then natural to ask: is it possible to find states that perform better? The answer to this question is positive [21]: there exist 'maximally multipartite entangled states' (MMES) such that their distribution function (over balanced bipartitions) is a Dirac delta function. For $n=2,3,5,6$ the delta is centered on the smallest possible value: $p\left(\pi_{A}\right)=\delta\left(\pi_{A}-1 / N_{A}\right)$. For $n=2$ MMES are Bell states up to local unitary transformations, while for $n=3$ MMES are equivalent to the GHZ states [22]. For $n=4$ one numerically obtains $p\left(\pi_{A}\right)=\delta\left(\pi_{A}-C_{4}\right)$, with $C_{4}=1 / 3>1 / 4=1 / N_{A}$ (but still $\left.C_{4}<1 / 2=2 / N_{A}\right)[21,23-25]$. For $n=7$ one finds states such that $p\left(\pi_{A}\right) \simeq \delta\left(\pi_{A}-C_{7}\right)$, with $C_{7} \simeq 0.136 \gtrsim 1 / 8=1 / N_{A}$. 'Perfect' MMES (that saturate the minimum purity $1 / N_{A}$ ) do not exist for $n \geqslant 8$ [26]. This will be presently viewed as a symptom of frustration and is 
a new and intriguing feature of the multipartite scenario, as opposed to the bipartite case. We shall now endeavor to outline a strategy that enables one to characterize MMES.

The search for MMES can be recast in terms of an optimization problem. Look at the average purity as a cost function ('potential of multipartite entanglement' [21])

$$
H=\mathbb{E}\left[\pi_{A}\right] \equiv\left(\begin{array}{c}
n \\
n_{A}
\end{array}\right)^{-1} \sum_{|A|=n_{A}} \pi_{A},
$$

where $\mathbb{E}$ denotes the expectation value and the sum is over balanced bipartitions $n_{A}=\lfloor n / 2\rfloor$. Since we are focusing on balanced bipartitions, and any bipartition can be brought into any other bipartition by applying a permutation of the qubits, the sum over balanced bipartitions in (7) is equivalent to a sum over the permutations of the qubits. $H$ in equation (7) is related to the linear entropy considered in [26-28].

The above minimization problem has proved to be of formidable difficulty, mainly for the presence of frustration: the requirement that purity saturates its minimum $1 / N_{A}$ can be satisfied for some but not for all balanced bipartitions. In other words, not all terms in the summation (7) can be $1 / N_{A}$. In order to study this problem, we will recast it in classical statistical mechanical terms: instead of searching for the minimum of $H$, one can look at the free energy of a suitable classical system at a fictitious temperature and recover the original problem in the zero temperature limit.

We follow the standard ensemble approach of statistical mechanics and introduce an ensemble $\left\{m_{j}\right\}$ of $M$ vectors (states), where $m_{j}$ is the number of vectors with purity $H=\epsilon_{j}$. One seeks the distribution that maximizes the number of states $\Omega=M ! / \prod_{j} m_{j}$ ! under the constraints that $\sum_{j} m_{j}=M$ and $\sum_{j} m_{j} \epsilon_{j}=M E$. For $M \rightarrow \infty$, the above optimization problem yields the canonical ensemble and its partition function

$$
Z(\beta)=\int \mathrm{d} \mu_{C}(z) \mathrm{e}^{-\beta H(z)}
$$

where the Lagrange multiplier $\beta$, that plays the role of an inverse temperature, fixes the average value of purity $E$. Note that the partition function (8) can be given a base-independent expression

$Z(\beta)=\int \mathrm{d} \mu_{C}(z) \mathrm{e}^{-\beta H(z)}=c_{N} \int \mathrm{d} \mu_{\mathrm{H}}(U) \exp \left(-\beta \mathbb{E}\left[\operatorname{tr}_{A}\left(\operatorname{tr}_{\bar{A}} U\left|\psi_{0}\right\rangle\left\langle\psi_{0}\right| U^{\dagger}\right)^{2}\right]\right)$,

where $\mu_{\mathrm{H}}$ denotes the Haar measure over $\mathcal{U}(\mathcal{H}),\left|\psi_{0}\right\rangle$ is any given vector and the (unimportant) constant $c_{N}=\mu_{C}\left(\mathbb{C}^{N}\right) / \mu_{\mathrm{H}}(\mathcal{U}(\mathcal{H}))$ is proportional to the ratio between the area of the $(N-1)$ dimensional sphere (3) and the volume of the unitary group.

The average over bipartitions in (7) entails a complicated dependence on the indices $j$ in equations (1)-(2). This hinders one from finding a closed expression for the partition function and makes the application of a standard saddle-point method or the calculation of the quenched average rather involved. Nonetheless, by applying the standard tools of statistical mechanics, one can discuss several aspects and analyze interesting limits. For $\beta \rightarrow 0$ equation (8) yields the distribution of the typical states (6). Note that this is valid both for $\beta \rightarrow 0^{ \pm}$. For $\beta \rightarrow+\infty$, only those configurations that minimize the Hamiltonian survive, namely the MMES. Remarkably, there is a physically appealing interpretation even for negative temperatures: for $\beta \rightarrow-\infty$, those configurations are selected that maximize the Hamiltonian, that is separable states. These preliminary findings are summarized in table 1. 
Table 1. High, low and negative temperature limits.

\begin{tabular}{lll}
\hline$\beta \rightarrow+\infty$ & $H=E_{0}(\min )$ & MMES \\
$\beta \rightarrow 0$ & $H \simeq \mu$ & Typical states \\
$\beta \rightarrow-\infty$ & $H=1(\max )$ & Separable states \\
\hline
\end{tabular}

By manipulating the partition function we can write the energy distribution function at arbitrary $\beta$ in terms of its high-temperature limit

$$
P_{\beta}(E)=\frac{1}{Z(\beta)} \int \mathrm{d} \mu_{C}(z) \delta(H-E) e^{-\beta H}=\frac{\mathrm{e}^{-\beta E} P_{0}(E)}{\int_{E_{0}}^{1} \mathrm{~d} E \mathrm{e}^{-\beta E} P_{0}(E)},
$$

where $E \in\left[E_{0}, 1\right], E_{0}$ being the minimum of the potential of multipartite entanglement $H$ in (7). We know that $1 / N_{A} \leqslant E_{0}\left(N_{A}\right) \leqslant \mu \leqslant 2 / N_{A}$, and thus $\lim _{N_{A} \rightarrow \infty} E_{0}\left(N_{A}\right)=0$. By multiplying and dividing the last equation by $|\beta| \mathrm{e}^{\beta}$ and $\beta \mathrm{e}^{\beta E_{0}}$, respectively, we find

$$
P_{-\infty}(E)=\delta(E-1), \quad P_{+\infty}(E)=\delta\left(E-E_{0}\right) .
$$

These limits are the counterparts of those discussed for the partition function and are reflected in the asymptotic behavior of the average energy as function of $\beta$

$$
\langle H\rangle_{\beta}=\frac{1}{Z(\beta)} \int \mathrm{d} \mu_{C}(z) H \mathrm{e}^{-\beta H}=\int_{E_{0}}^{1} \mathrm{~d} E E P_{\beta}(E)=-\frac{\partial}{\partial \beta} \ln Z(\beta) .
$$

Indeed,

$$
\langle H\rangle_{-\infty}=1, \quad\langle H\rangle_{+\infty}=E_{0} .
$$

Moreover $\frac{\partial}{\partial \beta}\langle H\rangle_{\beta}=-\left\langle H^{2}\right\rangle_{\beta}+\langle H\rangle_{\beta}^{2} \equiv-\triangle H_{\beta}^{2} \leqslant 0$, which is nonpositive. Thus the average energy is a non-increasing function of $\beta$ and has at least one inflexion point as function of $\beta$. From a qualitative point of view, one expects the behavior sketched in figure 2 : for $\beta \rightarrow 0$, the distribution is Gaussian (typical states); when $\beta \rightarrow+\infty$ the distribution tends to become more concentrated around $E_{0}$. This picture will be substantiated in the following.

In general, the high-temperature expansion of the average energy reads

$$
\begin{gathered}
\langle H\rangle_{\beta}=\sum_{m=1}^{\infty} \frac{(-\beta)^{m-1}}{(m-1) !} \kappa_{0}^{(m)}(H), \\
\kappa_{\beta}^{(m)}(H)=(-)^{m-1} \frac{\partial^{m-1}}{\partial \beta^{m-1}}\langle H\rangle_{\beta},
\end{gathered}
$$

where $\kappa_{\beta}^{(m)}(H)$ are the cumulants of $H$. So far, the analysis is valid at fixed $N=2^{n}$. Let us now briefly discuss the limit $N \rightarrow \infty$. The first cumulant is nothing but the average purity $\mu$ defined in (4). The second cumulant $\bar{\sigma}^{2}=\Delta H_{0}^{2}=\kappa_{0}^{(2)}(H)$ can also be computed exactly. A detailed calculation, to be presented elsewhere, yields

$$
\bar{\sigma}^{2}=\kappa_{0}^{(2)}(H) \sim 3 \sqrt{2} N^{-4+\log _{2} 3} \simeq O\left(N^{-2.42}\right) .
$$

The presence of an irrational exponent is a rather rare feature and an interesting phenomenon in itself. We note that if $\pi_{A}$ 's in sum (7) were independent Gaussian random variables, one would get $\bar{\sigma}^{2} \sim \sigma^{2} / N=O\left(N^{-3}\right)$, where $\sigma$ is defined in equation (5) (the number of balanced bipartitions being of order $N$.) This shows that different bipartitions 'interfere', yielding a highly nontrivial average. In fact, it can be shown that the correlations decrease exponentially with the distance between bipartitions. 


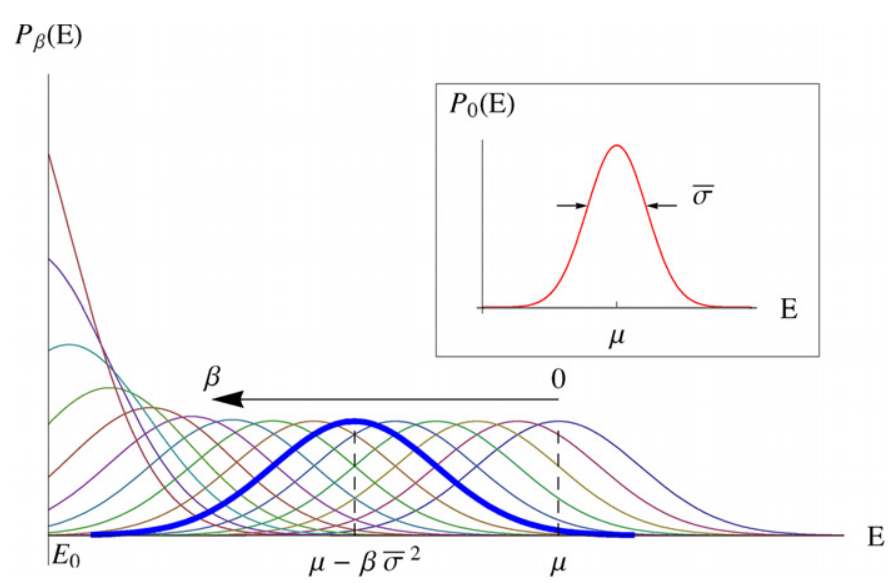

Figure 2. Qualitative sketch of equation (10), at fixed $N_{A}$, in arbitrary units. The energy density function is distributed around $\mu$ with standard deviation $\bar{\sigma}$ at $\beta=0$ (inset) and moves toward $E_{0}$ when $\beta \rightarrow+\infty$. From right to left, $\beta$ changes in constant steps. The probability density rigidly shifts with $\beta$, for $\beta \lesssim N^{7 / 2-\log _{2} 3}$. See equation (19) and following discussion. Note that $E_{0}=O\left(N^{-1 / 2}\right)$.

Higher order cumulants at $\beta=0$ can be shown to tend to zero faster than the others. Since the Gaussian is the only probability distribution with a finite number of non-vanishing cumulants (the first and the second one), one can therefore assume a Gaussian approximation for the energy distribution at infinite temperature, by neglecting all the cumulants but the first two

$$
P_{0}(E) \sim \frac{1}{\sqrt{2 \pi \bar{\sigma}^{2}}} \exp \left(-\frac{(E-\mu)^{2}}{2 \bar{\sigma}^{2}}\right),
$$

where $\mu$ and $\bar{\sigma}$ are defined in (4) and (16), respectively. Finally, by applying (10) we can compute the energy distribution at arbitrary temperature

$$
P_{\beta}(E) \sim \frac{1}{\sqrt{2 \pi \bar{\sigma}^{2}}} \exp \left(-\frac{\left(E-\mu+\beta \bar{\sigma}^{2}\right)^{2}}{2 \bar{\sigma}^{2}}\right) .
$$

Thus from definition (15) of cumulants, $\langle H\rangle_{\beta}$ as a function of $\beta$ would have a vanishing curvature, in contradiction with the qualitative sketch mentioned after equation (12). Equation (18) can be written as

$$
P_{\beta}(E)=P_{0}\left(E+\beta \bar{\sigma}^{2}\right),
$$

an expression valid also for non-Gaussian distributions that are well concentrated around their mean $\mu$. As a matter of fact, equations (18) and (19) are valid only when $\beta$ is not too large. In fact, they hold true as far as $\mu-\beta \bar{\sigma}^{2}-\bar{\sigma} \gtrsim 0$, i.e. $\beta \lesssim \mu / \bar{\sigma}^{2}=O\left(N^{7 / 2-\log _{2} 3}\right) \simeq O\left(N^{1.92}\right)$. Up to this value the probability density rigidly shifts with $\beta$, as is apparent in figure 2 . For larger values of $\beta$ the lower tail of the distribution starts 'feeling' the wall at $E_{0}$. The large- $\beta$ asymptotic form of $P_{\beta}(E)$ depends on the behavior of $P_{0}\left(E \rightarrow E_{0}\right)$ : one easily obtains

$$
P_{\beta}(E) \sim \frac{\beta^{\ell+1}}{\ell !}\left(E-E_{0}\right)^{\ell} \mathrm{e}^{-\beta\left(E-E_{0}\right)},
$$

where $\ell$ is the order of the first nonvanishing derivative of $P_{0}(E)$ at $E_{0}$. (Figure 2 displays the case $\ell=0$.) Note that the only relic of $P_{0}(E)$ in $(20)$ is $\ell$ and $P_{\beta \rightarrow+\infty}(E)$ yields the second equation in (11). The analysis for $\beta \rightarrow-\infty$ is analogous and yields the first equation in (11). 
In conclusion, we have built a statistical theory around an optimization problem, whose solutions are the maximally multipartite entangled states, that appear as minimal energy configurations. The approach we propose borrows methods from classical statistical mechanics in order to investigate the multipartite entanglement scenario. The introduction of a temperature is a familiar expedient in statistical mechanics, for instance in the study of optimization problems with simulated annealing and tempering. One obtains here an interesting picture for all real values of $\beta$ that fixes, with an uncertainty that becomes smaller for larger systems, the value of the purity of the subsystem under consideration, thus identifying an 'isoentangled' submanifold of states. A strategy similar to that adopted here was used in [29] for the simpler case of bipartite entanglement at a fixed bipartition, where the purity exhibits a phase transition. We have seen that the multipartite version of the problem is much more complicated, but it would be of great interest to understand whether the phase transition that occurs in the bipartite situation, when there is no average over the bipartitions, has a counterpart in the multipartite scenario. This would help elucidate the relation between multipartite entanglement, complexity and the presence of frustration.

\section{Acknowledgment}

This work is partly supported by the European Community through the Integrated Project EuroSQIP.

\section{References}

[1] Wootters W K 2001 Quantum Inf. Comp. 127

[2] Amico L, Fazio R, Osterloh A and Vedral V 2008 Rev. Mod. Phys. 80517

[3] Coffman V, Kundu J and Wootters W K 2000 Phys. Rev. A 61052306

[4] Wong A and Christensen N 2001 Phys. Rev. A 63044301

[5] Bruss D 2002 J. Math. Phys. 434237

[6] Meyer D A and Wallach N R 2002 J. Math. Phys. 434273

[7] Mezard M, Parisi G and Virasoro M A 1987 Spin Glass Theory and Beyond (Singapore: World Scientific)

[8] Man'ko V I, Marmo G, Sudarshan E C G and Zaccaria F 2002 J. Phys. A: Math. Gen. 357137

[9] Facchi P, Florio G and Pascazio S 2006 Phys. Rev. A 74042331

[10] Facchi P, Florio G and Pascazio S 2007 Int. J. Quantum Inf. 597

[11] Horodecki M, Horodecki P and Horodecki R 1998 Phys. Rev. Lett. 805239

[12] Bennett C H, DiVincenzo D P, Mor T, Shor P W, Smolin J A and Terhal B M 1999 Phys. Rev. Lett. 825385

[13] Meja-Monasterio C, Benenti G, Carlo G G and Casati G 2005 Phys. Rev. A 71062324

[14] Rossini D and Benenti G 2008 Phys. Rev. Lett. 100060501

[15] Lubkin E 1978 J. Math. Phys. 191028

[16] Lloyd S and Pagels H 1988 Ann. Phys., N.Y. 188186

[17] Page D N 1993 Phys. Rev. Lett. 711291

[18] Zyczkowski K and Sommers H-J 2001 J. Phys. A: Math. Gen. 347111

[19] Scott A J and Caves C M 2003 J. Phys. A: Math. Gen. 369553

[20] Giraud O 2007 J. Phys. A: Math. Theor. 402793

[21] Facchi P, Florio G, Parisi G and Pascazio S 2008 Phys. Rev. A 77060304

[22] Greenberger D M, Horne M and Zeilinger A 1990 Am. J. Phys. 581131

[23] Higuchi A and Sudbery A 2000 Phys. Lett. A 273213

[24] Brown I D K, Stepney S, Sudbery A and Braunstein S L 2005 J. Phys. A: Math. Gen. 381119

[25] Brierley S and Higuchi A 2007 J. Phys. A: Math. Theor. 408455

[26] Scott A J 2004 Phys. Rev. A 69052330

[27] Emary C 2004 J. Phys. A: Math. Gen. 378293

[28] Rigolin G, de Oliveira T R and de Oliveira M C 2006 Phys. Rev. A 74022314

[29] Facchi P, Marzolino U, Parisi G, Pascazio S and Scardicchio A 2008 Phys. Rev. Lett. 101050502 